\title{
COL17A1 germline variant p.Ser1029Ala and mucosal malignant melanoma: An autopsy study
}

\author{
DAIKE TONG $^{1}$, MASASHI TANAKA ${ }^{2}$, HIDETAKA EGUCHI ${ }^{3}$, YASUSHI OKAZAKI ${ }^{3}$, \\ MASAAKI MURAMATSU ${ }^{1}$ and TOMIO ARAI ${ }^{4}$
}

\begin{abstract}
${ }^{1}$ Department of Molecular Epidemiology, Medical Research Institute, Tokyo Medical and Dental University, Tokyo 113-8510;
${ }^{2}$ Department of Neurology, Graduate School of Medicine, Juntendo University; ${ }^{3}$ Diagnostics and Therapeutics of Intractable Diseases, Intractable Disease Research Center, Graduate School of Medicine, Juntendo University, Tokyo 113-8421; ${ }^{4}$ Department of Pathology, Tokyo Metropolitan Geriatric Hospital, Tokyo 173-0015, Japan
\end{abstract}

Received September 2, 2021; Accepted November 4, 2021

DOI: $10.3892 / \mathrm{mco} .2021 .2465$

\begin{abstract}
Collagen type XVII $\alpha 1$ (COL17A1) encodes a hemidesmosomal protein at the epidermal-dermal junction and its variants are implicated in blistering skin diseases. Recent experiments in rodents revealed that Col17a1 has critical roles in stem cells of epidermal origin and in melanoma carcinogenesis. In the present study, it was investigated whether germline variants in COL17A1 are associated with skin cancer and other cancer types using indexed consecutive autopsy cases from the Japanese Geriatric Single Nucleotide Polymorphism database $(n=2,343$; mean age, 80 years). The database included 12 patients with skin cancer. A total of 53 COL17A1 missense variants on an exome chip were analyzed. One variant, p.Ser1029Ala (rs118166857), which had a minor allele frequency of $1.0 \%$, exhibited a nominal positive sign of association with skin cancer [Fisher's exact $\mathrm{P}=0.002$, odds ratio $(\mathrm{OR})=16.93,95 \% \mathrm{CI}$ : 4.44-64.64]. This variant was detected in $2 / 2$ patients with mucosal malignant melanoma (mMM) and $1 / 3$ patients with extramammary Paget's disease, and in none of the patients with non-melanoma cancer, e.g., squamous cell and basal cell carcinoma. Other cancer types were searched in the database and the p.Ser1029Ala variant was indicated to be nominally associated with breast cancer $(\mathrm{P}=0.006, \mathrm{OR}=4.17$, 95\% CI: 1.72-10.11). In the two mMM cases, targeted exome sequencing of 55 cancer-predisposing genes (including tumor protein 53, BRCA1/2 and mismatch repair genes) detected no apparent pathogenic variants, but revealed variants of unknown significance in axin 2, DNA directed polymerase $\zeta$ catalytic subunit and contactin 6 . Since COL17A1 provides a niche for melanocyte stem cells, it was hypothesized that the p.Ser1029Ala variant in the COL17A1 ectodomain may affect the microenvironment, e.g., the cell competition. This is a working
\end{abstract}

Correspondence to: Professor Masaaki Muramatsu, Department of Molecular Epidemiology, Medical Research Institute, Tokyo Medical and Dental University, 1-5-45 Yushima, Bunkyo-ku, Tokyo 113-8510, Japan

E-mail: muramatsu.epi@mri.tmd.ac.jp

Key words: COL17, BP180, cancer-predisposing, rare variant hypothesis generated from human autopsy cases and warrants further epidemiological and molecular biological validation.

\section{Introduction}

Malignant melanoma ( $\mathrm{MM})$ is a rare refractory tumor that originates from melanocyte stem cells and is associated with aggressive behavior and poor prognosis. MM is divided into two categories according to the primary lesions, mucosal malignant melanoma (mMM) and cutaneous malignant melanoma (cMM) $(1,2)$, which differ in epidemiology and etiology. While cMM is more common among Caucasians, mMM is relatively more common among Asians. Ultraviolet light exposure is a major risk factor for cMM but not for mMM, and mMM develops in later life stages than cMM. Investigation of $\mathrm{mMM}$ has been difficult due to the rarity of this disease and little is known about its pathogenesis and genetics. Understanding the physiology of melanocyte stem cells may be central to elucidating the carcinogenesis of MM.

The transmembrane protein collagen type XVII (COL17) is mainly expressed in epidermal basal keratinocytes and is a structural component of the hemidesmosome, an adhesion complex at the epidermal-dermal junction. COL17 is involved in skin diseases, with germline mutations in COL17 resulting in junctional epidermolysis bullosa (JEB) and autoimmunity towards COL17 leading to bullous pemphigoid (3). Altered COL17 expression has been reported in various skin cancer types, including melanoma (4). Recent experiments in rodent models have revealed that a truncation mutation in the Col17a1 gene promoted melanoma progression (5).

In the present study, COL17 $\alpha 1$ (COL17A1) was selected as a candidate gene and it was examined whether its variants were related to skin cancers and other cancer types using catalogued autopsy cases in the Japanese Geriatric Single Nucleotide Polymorphism (JG-SNP) database.

\section{Materials and methods}

Study population. The present study included subjects registered in the JG-SNP (6), which comprised consecutive autopsy cases collected at Tokyo Metropolitan Geriatric 
Hospital (Tokyo, Japan) between 1995 and 2012. Autopsy procedures were performed on $\sim 29 \%$ of the patients who died in the hospital. The JG-SNP included a total of 2,343 subjects (1,298 males and 1,045 females). The mean age at the time of death was $80.6 \pm 8.8$ years. The presence or absence of any disease was determined by a thorough examination upon autopsy. Details of the JG-SNP database are described elsewhere (6). The distributions of diseases in the study group did not substantially differ from those reported in a survey by the Ministry of Health, Labor and Welfare of Japan (7). For panel sequencing, two cases of $\mathrm{mMM}$ were selected.

Genotyping and statistical analysis. Genomic DNA was extracted from the renal cortex using a standard phenol-chloroform procedure and kept at $-80^{\circ} \mathrm{C}$ until use. All samples were genotyped using the Infinium Human Exome Bead Chip Version 1.1 (Illumina, Inc.) by iScan, in accordance with the supplier's protocol. Genotype calling was performed for all samples, as a single project using the Genotyping Module (version 1.9) of the GenomeStudio data analysis software package. Initial genotype clustering was performed using the default Illumina cluster file (HumanExome 12v1-1_A.egt) and manifest file (HumanExome-12v1-1_A.bpm), applying the GenTrain 2 clustering algorithm. Specimens with a per-sample call rate of $>98 \%$ were considered eligible. Associations between the variants and cancer status of the patients were displayed on a contingency table and Fisher's exact tests were performed by using SPSS Statistics 25.0 (IBM Corporation). To minimize bias, the pathological assessment, genotyping and statistical analysis had been performed at different institutions in a double-blinded manner.

Panel sequencing. A total of two cases of mMM were selected for panel sequencing analysis. AmpliSeq 7.0.2 (Thermo Fisher Scientific, Inc.) was used to design an AmpliSeq Custom panel that covers the exons, exon-intron boundaries and 5'UTR and 3'UTR of the genes encoding AKT1, APC, ATM, AXIN2, BMPR1A, BRCA1, BRCA2, BARD1, BRIP1, BUB1, BUB1B, BUB3, CDH1, CDKN1B, CHEK2, contactin 6 (CNTN6), ENG, EPCAM, EPHX1, FANCC, FANCE, FAN1, GALNT12, LRP6, MBD4, MCM9, MYH11, MLH1, MLH3, MSH2, MSH3, MSH6, MUTYH, NFKBIZ, NTHL1, PIK3CA, PMS1, PMS2, POLD1, POLE, POLQ, PTEN, RAD52, RBL1, REV3 like, DNA directed polymerase $\zeta$ catalytic subunit (REV3L), RNF43, RPS20, SCG5-GREM1, SDHB, SDHD, SMAD4, SMAD9, SMARCA4, STK11, TDRD3, TGFBR2, TP53, UIMC1, XAF1 and XRCC4, as well as the upstream regions of the genes encoding GREM1, APC and MSH2 (8). An Ion Chef instrument was used for emulsion PCR, bead enrichment and chip loading onto the Ion 530 chip (Thermo Fisher Scientific, Inc) using the Ion 520 and 530 kits (Thermo Fisher Scientific, Inc.). The loaded chips were subjected to sequencing on an Ion GeneStudio S5 Plus sequencer (Thermo Fisher Scientific, Inc.). The obtained data were analyzed using Ion Reporter server 5.10 (Thermo Fisher Scientific, Inc.). The called variants were reviewed by visual inspection on the Integrative Genomics Viewer (version 4.2, 15 September 2009; Broad Institute).

Only variants including missense, nonsense, indel and splice site variants were retained. Variants with a minor allele frequency (MAF) of $<1 \%$ according to the JMorp database, which catalogues variations in $8.6 \mathrm{~K}$ Japanese individuals (9), were selected. The mutation databases of InterVar (https://wintervar.wglab.org/) and the Human Gene Mutation Database (http://www.hgmd.cf.ac.uk/ac/index.php) professional version as of April 2021 were used to annotate variants as pathogenic, likely pathogenic, of unknown significance, likely benign or benign. Polyphen- 2 was used to predict the functional consequence of the amino acid substitution (10).

\section{Results}

Exome chip analysis. The JG-SNP database included a total of 12 patients with skin cancer, comprising two with $\mathrm{mMM}$ (81 years, male; 83 years, male), three with extramammary Paget's disease (EMPD) (93 years, female; 91 years, male; 76 years, male), three with squamous cell carcinoma (SCC) ( 85 years, male; 87 years, female; 92 years, female), two with basal cell carcinoma (BCC) (77 years, female; 92 years, female), one with apocrine carcinoma ( 83 years, male) and one with unknown histology origin (90 years, male). The database included no cases of cMM.

Fig. 1 presents a flow chart of the analysis to determine germ line variants in the candidate COL17A1 gene. Altogether, 53 COL17A1 missense single nucleotide variants (SNVs) were analyzed on the Exome Chip, of which 52 were successfully genotyped. Analysis of 2,343 subjects revealed that 40 SNVs were monomorphic and 12 were bimorphic. These SNVs were analyzed with regard to association with skin cancer and other cancer types. Only one nonsynonymous variant, p.Ser1029Ala (rs118166857), exhibited a nominal sign of association with skin cancer [Fisher's exact $\mathrm{P}=0.002$; odds ratio $(\mathrm{OR})=16.93$, 95\% CI: 4.44-64.64; Table I]. The other 11 variants did not exhibit any positive sign of association (data not shown).

It was indicated that the p.Ser1029Ala variant was carried by two of the two patients with mMM and one of the three patients with EMPD, but not by any of the patients with other skin cancers, including SCC and BCC. Among the two cases of mMM, case one was an 81-year-old male who had mMM that originated in the anal canal, with massive metastasis to the lymph nodes and brain. The tumor cells exhibited melanin granules and immunohistochemistry revealed positive staining for S100A and HMB-45 (anti-melanoma). Case two was an 83-year-old male with a large lung tumor with necrosis in the center, as well as brain metastasis, both of which were histologically confirmed as MM. Close inspection for the primary lesion at the skin, esophagus, oral cavity, pharynx, larynx, trachea, anus and external genital organs did not reveal any relevant lesions. Thus, this case was most likely rare lung MM (11), although it was not possible to exclude the possibility of MM with an undetected or diminished primary nest.

The database included three cases of EMPD. The one patient with EMPD who carried the p.Ser1029Ala variant was a 76-year-old male with lesions at the external genital organs. This case exhibited infiltration in the epidermis without distant metastasis.

The p.Ser1029Ala variant appeared in 47 heterozygote carriers and one homozygote and the MAF was $1.0 \%$. These 48 carriers were surveyed for other cancers (Table I) and it was indicated that most cancers were not associated with 


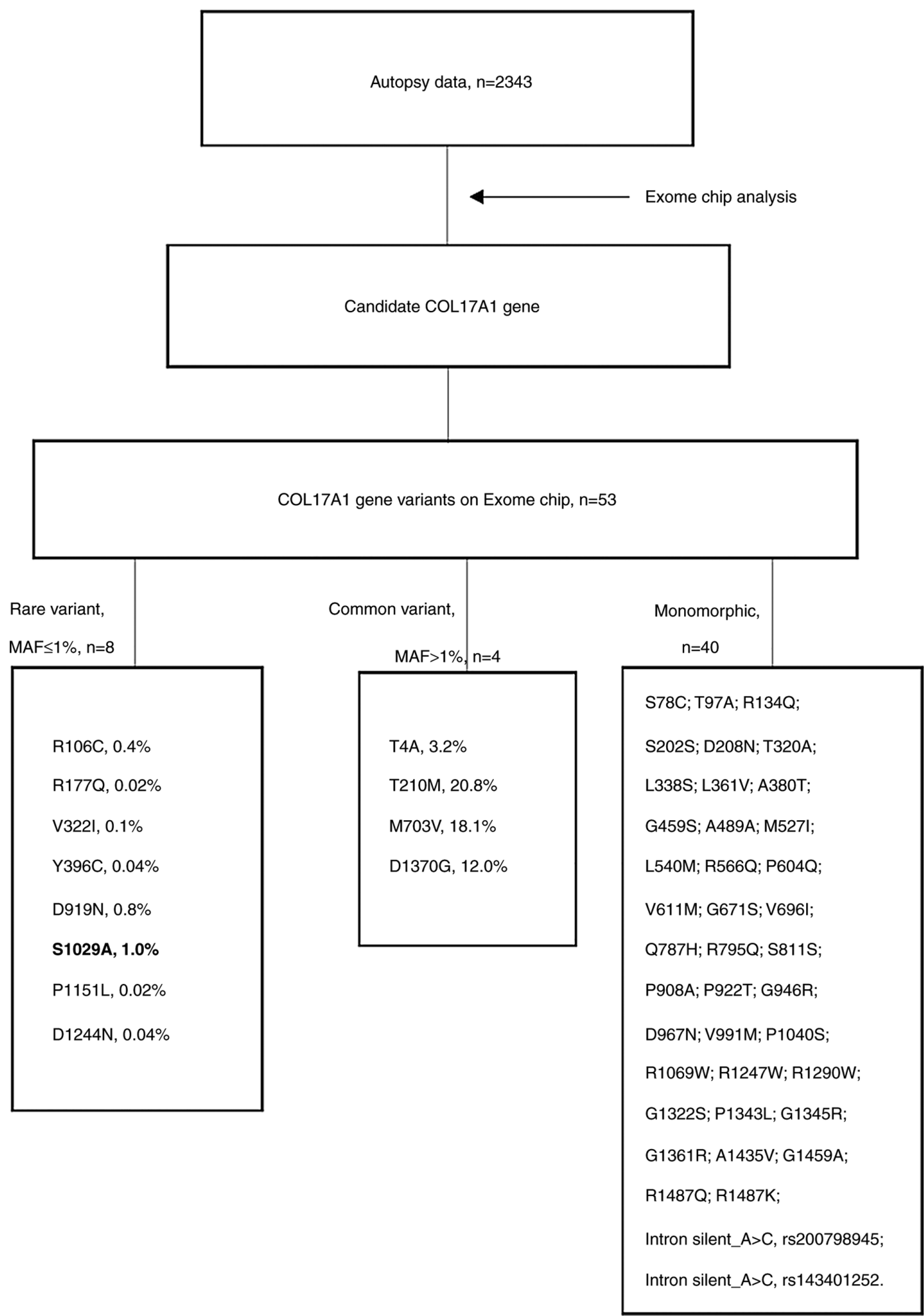

Figure 1. Flow chart of COL17A1 variant analysis in the Japanese Geriatric Single Nucleotide Polymorphism database. MAF, minor allele frequency; COL17A1, collagen type XVII $\alpha 1$.

this variant. However, a nominal positive sign of association with breast cancer was obtained $(\mathrm{P}=0.006, \mathrm{OR}=4.17,95 \% \mathrm{CI}$ : 1.72-10.11).

Targeting sequencing of two mMM cases. To discover further potential germline variants, panel sequencing of 55 cancer-predisposing genes (including BRCA1/2, TP53 and mismatch repair genes) was performed in the two patients with mMM. Among the 55 genes, 404 variants were identified in case 1 and 489 variants in case 2. These comprised both common and rare variants and included single-nucleotide and multi-nucleotide variants, as well as insertion/deletions. From the pathogenic variants, the synonymous variant types and unknown types were removed. Finally, 33 missense variants were identified in case 1 and 41 missense variants in case 2 , including both common and rare variants (Fig. 2). 
Table I. Characteristics of p.Ser1029Ala carriers.

\begin{tabular}{|c|c|c|c|c|}
\hline Characteristic & $\begin{array}{l}\text { p.Ser1029Ala }(+) \\
(\mathrm{n}=48)\end{array}$ & $\begin{array}{l}\text { p.Ser1029Ala (-) } \\
\quad(n=2,295)\end{array}$ & P-value & OR $(95 \% \mathrm{CI})$ \\
\hline Sex, F/M & $22 / 26$ & $1023 / 1272$ & 0.884 & $1.05(0.59-1.87)$ \\
\hline Age, years & $82.0 \pm 9.2$ & $80.6 \pm 8.8$ & $0.217^{\mathrm{a}}$ & $\mathrm{NC}$ \\
\hline Total cancer, +/- & $34 / 14$ & $1412 / 883$ & 0.230 & $1.52(0.81-2.85)$ \\
\hline Skin cancer & 3 & 9 & 0.002 & $16.93(4.44-64.64)$ \\
\hline Mucosal melanoma & 2 & 0 & 0.001 & $\mathrm{NC}$ \\
\hline Paget's disease & 1 & 2 & 0.060 & $24.39(2.17-273.70)$ \\
\hline Other skin cancer & 0 & 7 & 0.997 & $1(0.99-1.00)$ \\
\hline Blood cancer & 7 & 205 & 0.197 & $1.74(0.77-3.93)$ \\
\hline Breast cancer & 6 & 76 & 0.006 & $4.17(1.72-10.11)$ \\
\hline Colorectal cancer & 6 & 389 & 0.559 & $0.7(0.3-1.66)$ \\
\hline Myelodysplastic syndrome & 3 & 39 & 0.053 & $3.86(1.15-12.94)$ \\
\hline Urinary tract cancer & 3 & 43 & 0.066 & $3.49(1.04-11.67)$ \\
\hline Prostatic cancer & 3 & 210 & 0.798 & $0.66(0.20-2.15)$ \\
\hline Gastric cancer & 3 & 259 & 0.358 & $0.52(0.16-1.70)$ \\
\hline Malignant lymphoma & 3 & 122 & 0.740 & $1.19(0.36-3.88)$ \\
\hline Thyroid cancer & 2 & 52 & 0.304 & $1.88(0.44-7.93)$ \\
\hline Lung cancer & 2 & 272 & 0.114 & $0.32(0.08-1.34)$ \\
\hline Biliary tract cancer & 2 & 62 & 0.380 & $1.57(0.37-6.60)$ \\
\hline Esophageal cancer & 1 & 31 & 0.487 & $1.55(0.21-11.62)$ \\
\hline Mesothelioma & 1 & 4 & 0.098 & $12.19(1.34-111.10)$ \\
\hline Myeloma & 1 & 39 & 0.566 & $1.23(0.17-9.15)$ \\
\hline Small intestine cancer & 1 & 11 & 0.220 & $4.42(0.56-34.92)$ \\
\hline Kidney cancer & 1 & 33 & 0.508 & $1.46(1.20-10.89)$ \\
\hline
\end{tabular}

Values are expressed as $\mathrm{n}$ or the mean \pm standard deviation. ${ }^{a}$ Result based on T-test; all other P-values are according to Fisher's test. OR, odds ratio; $\mathrm{CI}$, confidence interval; $\mathrm{F}$, female; $\mathrm{M}$, male; $\mathrm{NC}$, non-conformance.

Table II presents the rare variants, which had MAF values of $<1 \%$ according to the database of ToMMo $8.3 \mathrm{KJPN}$ (https://jmorp.megabank.tohoku.ac.jp/202102/). There were 4 rare variants in case 1 (REV3L p.His1319Tyr, $\mathrm{MAF}=0.14 \%$; BRCA1 p.Tyr856His, MAF=0.78\%; BUB1 p.Cys698Tyr, $\mathrm{MAF}=0.13 \%$; NFKBIZ p.Thr307Ser, $0.75 \%$ ) and 4 rare variants in case 2 (CNTN6 p.Arg471Thr, MAF=0.67\%; AXIN2 p.His513Tyr MAF=0\%; REV3L p.Arg1970His, $\mathrm{MAF}=0.85 \%$; ATM p.His683Gln, MAF=0\%). The AXIN2 p.His513Tyr and ATM p.His683Gln variants were novel variants without accession numbers in the GenBank database (https://www.ncbi.nlm. nih.gov/genbank/).

InterVar and Polyphen-2 were used to annotate the pathogenicity of the variants. Variants of unknown significance included REV3L p.His1319Tyr and BUB1 p.Cys698Tyr in case 1, and CNTN6 p.Arg471Thr, AXIN2 p.His513Tyr and REV3L p.Arg1970His in case 2. The functional significance of variants was predicted using Polyphen-2 and three possibly damaging predictions and one probably damaging prediction were obtained. In case 1 , the Polyphen score was 0.828 for REV3L p.His1319Tyr and 0.730 for BRCA1 p.Tyr856His, although the InterVar results indicated benign features. The Polyphen score was 0.007 for BUB1 p.Cys698Tyr and 0 for NFKBIZ p.Thr307Ser. In case 2, the Polyphen score was 0.918 for CNTN6 p.Arg471Thr, 1 for the novel variant AXIN2
p.His513Tyr, 0.002 for REV3L p.Arg1970His and 0.002 for ATM p.His683Gln.

\section{Discussion}

A nationwide registry of MM in Japan included 5,566 patients with MM during the three years of 2011-2013 (12). Among these patients, one in seven had mMM. In Japan, MM is a rare cancer type and $\mathrm{mMM}$ accounts for a higher proportion of MM cases compared to that in Caucasians (13). In mMM cases, lesions are most common in the head and neck (51.6\%), followed by the gastrointestinal tract $(28.3 \%)$. Primary mMM in the lung is rare, accounting for only $1.7 \%$ of mMM (12). Thus, the patients analyzed in the present study were rare refractory cancer cases. The two melanoma patients in the JG-SNP database had the mucosal rather than the cutaneous type. The expression of col17 is $\sim 30-50 \%$ higher in mucosal keratinocytes than in skin keratinocytes, suggesting that col17 may also have an important role in mucosal keratinocytes (14).

In the present study, a rare missense variant, COL17A1 p.Ser1029Ala $(\mathrm{MAF}=1.0 \%)$, was detected in two of the two patients with $\mathrm{mMM}$ and one of the three patients with EMPD. This variant was not observed in SCC and BCC patients. This may be explained by the notion that the pathogenesis of non-melanoma skin cancers predominantly involves other 
Table II. Rare variants in patients with mucosal melanoma.

A, Patient 1

\begin{tabular}{|c|c|c|c|c|c|c|}
\hline Gene & SNV & VE & dbSNP & InterVar & Prediction $^{\mathrm{a}}$ & $\operatorname{MAF}^{\mathrm{b}}(\%)$ \\
\hline REV3L & p.His1319Tyr & Missense & rs763112147 & US & Possibly damaging & 0.14 \\
\hline BRCA1 & p.Tyr856His & Missense & rs80356892 & $\mathrm{B}$ & Possibly damaging & 0.78 \\
\hline BUB1 & p.Cys698Tyr & Missense & rs764154158 & US & Benign & 0.13 \\
\hline NFKBIZ & p.Thr307Ser & Missense & rs3821727 & LB & Benign & 0.75 \\
\hline
\end{tabular}

B, Patient 2

\begin{tabular}{|c|c|c|c|c|c|c|}
\hline Gene & SNV & VE & dbSNP & InterVar & Prediction $^{\mathrm{a}}$ & $\operatorname{MAF}^{\mathrm{b}}(\%)$ \\
\hline CNTN6 & p.Arg471Thr & Missense & rs763596062 & US & Possibly damaging & 0.67 \\
\hline AXIN2 & p.His513Tyr & Missense & NV & US & Probably damaging & 0 \\
\hline REV3L & p.Arg 1970 His & Missense & rs3218606 & US & Benign & 0.85 \\
\hline ATM & p.His683Gln & Missense & NV & LB & Benign & 0 \\
\hline
\end{tabular}

${ }^{a}$ Results based on polyphen-2. ${ }^{b}$ MAF according to jMorp (Tommo8.3k). NV, novel variant; SNV, single nucleotide variant; VE, variant effect; MAF, minor allele frequency; US, uncertain significance; LB, likely benign; B, benign.

adhesion molecules, such as cadherins, integrins and selectins (15). It was also observed that the COL17A1 p.Ser1029Ala variant was nominally associated with breast cancer. Considering the recent findings that Col17A1 functions as an essential molecule for the stem cell niche and cell competition $(16,17)$, this variant may be worth studying for its potential cancer-predisposing role.

COL17A1 is one of the triple helical collagen genes, which encodes a type 2 oriented transmembrane protein with collagenous domains in the extracellular domain. COL17A1 is a component of the hemidesmosome, a multi-protein adhesion complex that maintains stable attachment at the dermal-epidermal junction. COL17A1 is well known as a causative gene for JEB, which leads to fragile-desmosome and skin-blistering phenotypes (18). Over 40 rare variants of COL17A1 have been reported [Human Genome Mutation Database professional (http://www.hgmd.cf.ac.uk/ac/index. php), as of April 2021]. Most are nonsense variants, while certain variants are missense variants, which are generally associated with milder phenotypes, such as more localized lesions and late disease onset. This list does not include the p.Ser1029Ala mutation identified in the present study. This variant is c.3085 $\mathrm{T}>\mathrm{G}$ on the COL17A1 cDNA. It is located on exon 45 and the residue resides in the non-collagen (NC) 10 region in the ectodomain. Functional prediction using polyphen- 2 and InterVar suggested that the consequence of amino acid substitution at this position is benign, indicating that it is not a null functional mutation.

COL17A1 expression is upregulated in association with the invasion of melanoma (4) and its role in early carcinogenesis was recently uncovered. In a mouse model, Col17a1 dysfunction, caused by the introduction of $\mathrm{NC16}$ deletion, supported melanoma progression through modulation of the skin tumor microenvironment by basal keratinocytes (5). Col17a1 is not expressed in melanocytes but is expressed in adjacent kerati- nocytes and provides a niche for melanocyte stem cells (16). Col17a1 is dynamically shed by proteases at the NC16A domain, which has an important role in cell motility, adhesion and cell differentiation at the epidermal site. It is tempting to speculate that the variant p.Ser1029Ala in the ectodomain may be less efficiently shed compared to the wild-type, thereby assisting melanoma progression (Fig. 3).

Recent results obtained with Col17a1-null mice revealed that this protein functions as an essential molecule for the proliferation of melanocyte stem cells and has essential roles in cell development, aging and carcinogenesis at epidermal sites (19). These results appear to be in line with the present observation that p.Ser1029Ala of COL17A1 may have a role in melanoma progression (5). COL17A1 has recently been demonstrated to have an important role in cell competition (17), a newly described mechanism that allows tumor cells to outcompete and eliminate less fit adjacent normal cells (20). It is tempting to speculate that p.Ser1029Ala in the ectodomain may change the microenvironment to favor normal cells being easily outcompeted.

A potential link between COL17A1 and breast cancer has been suggested by findings that COL17A1 expression is suppressed in breast cancer cells (21) and that replenishing COL17A1 expression has an anti-proliferative effect (22). In breast cancer cells, COL17A1 participates in regulating the transition from monolayered to multilayered epithelial cells in precancerous lesions. This may be related to its role in tumorigenesis of the monolayered epithelia of the mammary gland (23).

Although the two patients with mMM in the present study had no familial history, other potential cancer-predisposing genes and variants were searched by examining the sequences of 55 cancer-related genes. No pathogenic germline mutations in BRCA1/2 or P53 were identified, but only variants of unknown significance were found in genes possibly related to melanoma. 


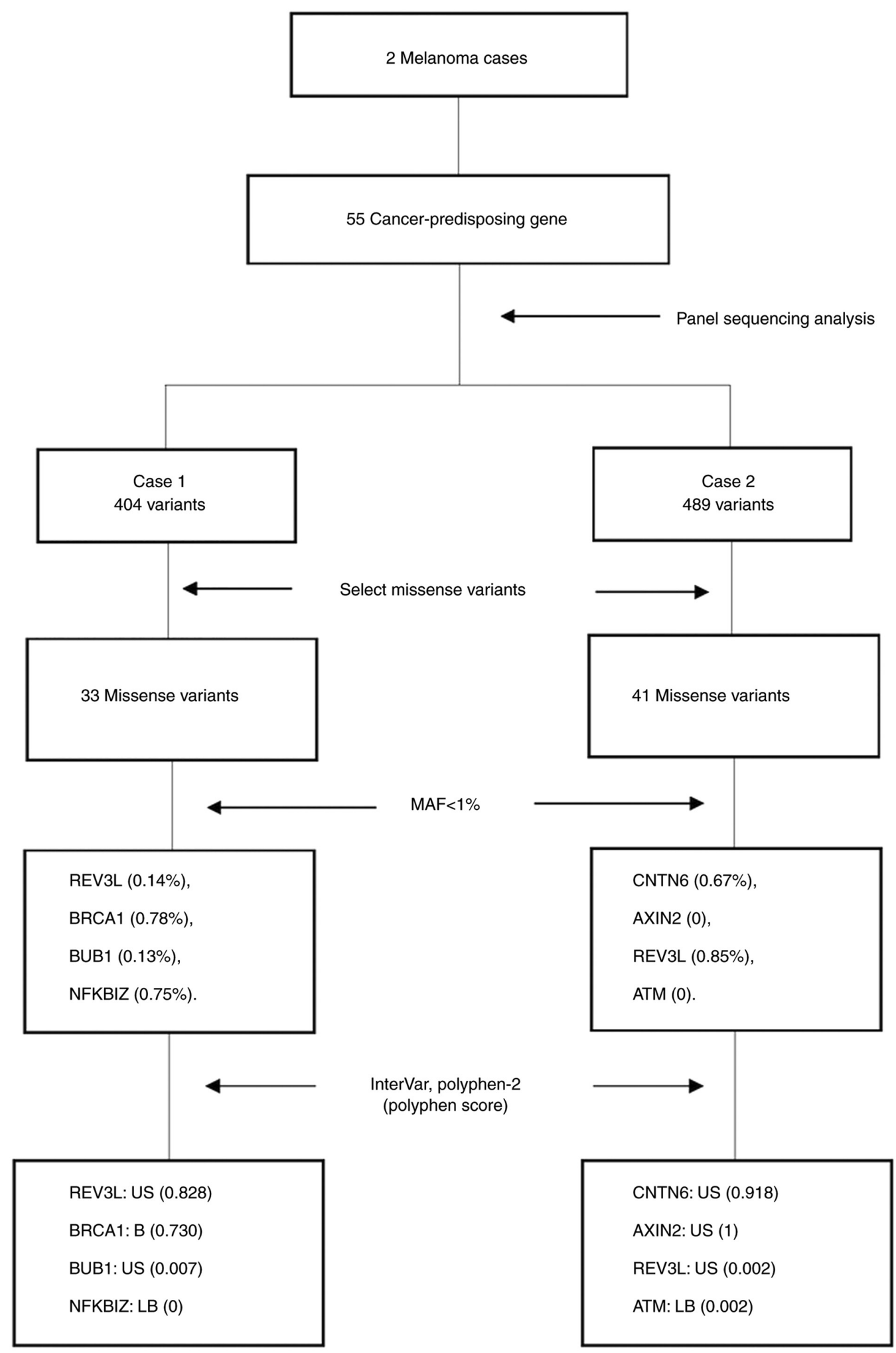

Figure 2. Flow chart for exome panel sequencing of 55 genes in two cases of mucosal malignant melanoma. US, uncertain significance; B, benign; LB, like benign; MAF, minor allele frequency.

REV3L, also known as DNA polymerase $\zeta$, is the largest catalytic subunit of DNA polymerase and is important in translation synthesis, which is usually the first line of defense against ultraviolet radiation or chemical adducts in DNA (24). Somatic mutation of REV3L is frequently identified in cancer, including melanoma (25). In melanoma cells, REV3L constitutes an important part of the response to cisplatin, resulting in improved survival and growth (26). Of note, REV3L also binds and is down-regulated by microRNA (miR)-340 (27), which is a modulator of RAS-RAF-MAPK signaling in melanoma (28). It may be worthwhile investigating the mutual regulation between REV3L and miR-340 in melanoma.

AXIN2 acts as a negative regulator in the Wnt signaling pathway. It forms a complex with GSK-3 $\beta$ and $\beta$-catenin and 

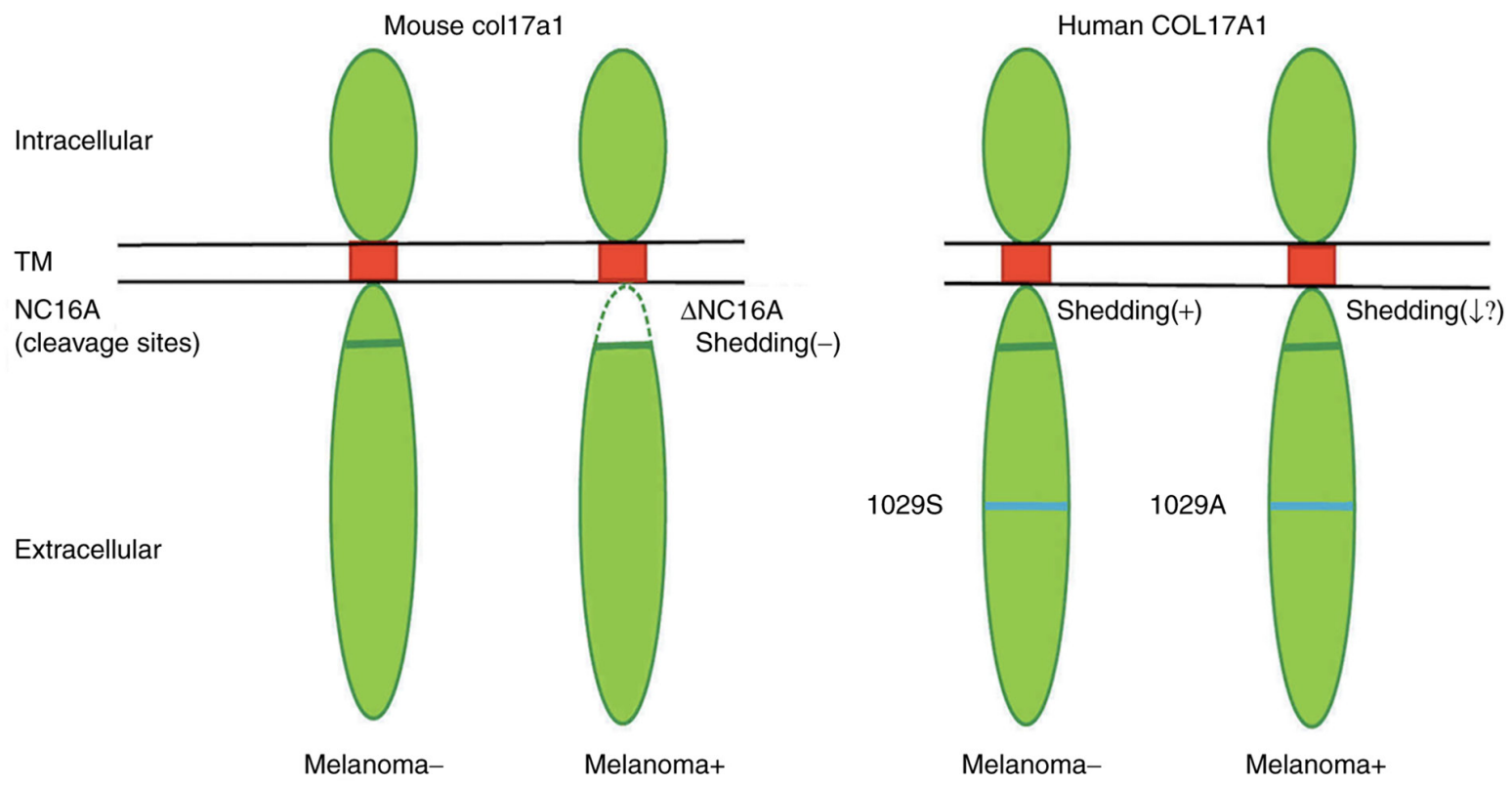

Figure 3. A working hypothesis for COL17A1 variants and melanoma. COL17A1, collagen type XVII $\alpha 1$; NC16, non-collagen 16; TM, transmembrane.

promotes GSK-3 $\beta$-dependent phosphorylation of $\beta$-catenin. The novel AXIN2 p.His513Tyr variant identified in the present study appears to be localized at the GSK-3 $\beta$ interaction site $(29,30)$. Wnt/ $\beta$-catenin dysregulation is the key to melanocyte deterioration. The melanoma cell line PR-Mel carries an AXIN2 deletion mutation (31). Specifically, AXIN2 is a negative regulator of $\mathrm{Wnt} / \beta$-catenin signaling, which acts to stimulate hair growth in the hair follicle stem cell (32). Loss of function of Drosophila Axn may cause cell competition by inducing cell death in the surrounding wild-type cells (19). Thus, an AXIN2 variant may have a fundamental role in melanoma.

CNTN6 is a ligand of NOTCH receptor 1 (NOTCH1). The NOTCH1 signaling pathway is a determinant of stemness and plasticity in melanoma stem/initiating cells, through regulation of melanoma aggressiveness (33). NOTCH1 is also involved in cell competition (19). BUB1 is related to melanoma, in that BUB1 expression distinguishes melanoma, benign nevi and lymph nodes, thus contributing to the detection of melanoma micrometastasis in sentinel lymph nodes. High BUB1 expression is found in metastatic melanoma. BUB1 is downstream of sirtuin 1, which is upregulated in melanoma and its inhibition exerts an anti-proliferative effect in melanoma cells (34). The rare variants found in these genes may relate to melanoma predisposition by affecting cell competition.

The present study has certain limitations. Consecutive autopsy cases from geriatric hospitals were used as cancer and control subjects. Obviously, there is a survival bias due to the high age of the subjects. Indeed, this sample does not represent subjects who died prematurely. Due to being an autopsy study, the present study was also limited in terms of access to clinical data related to cancer progression and tumor stages. Relevant pathological samples to perform an immunohistochemical analysis are not available, so that it is not possible to determine whether the p.Ser1029Ala variant affected the shedding of COL17A1. The small sample size for melanoma may be a hindrance to determining a significant association.
In conclusion, the present study reported on a COL17A1 p.Ser1029Ala variant identified in rare malignancies of epithelial origin in an autopsy database. The present study was retrospective and the statistical analyses were based on a small number of patients; thus, the present observations only serve for hypothesis generation. There remains a requirement for further studies including larger numbers of patients and molecular pathogenesis.

\section{Acknowledgements}

Not applicable.

\section{Funding}

This study was funded by NANKEN KYOTEN TMDU.

\section{Availability of data and materials}

The datasets used and/or analyzed during the present study are available from the corresponding author on reasonable request.

\section{Authors' contributions}

MM designed the present study. TA performed the pathological analysis. MT acquired and analysed clinical data. HE and YO generated the targeting sequencing data. DT performed genotyping and statistical analyses. MM, TA and MT confirm the authenticity of all the raw data. DT and MM wrote the manuscript. All authors read and approved the manuscript.

\section{Ethics approval and consent to participate}

This study was approved by the Ethics Committees of Tokyo Medical and Dental University (Tokyo, Japan; approval no. 02016-011-03) and the Tokyo Metropolitan Geriatric Hospital (Tokyo, Japan; approval no. 230405). 
Written informed consent was obtained from a family member of each study participant.

\section{Patient consent for publication}

Not applicable.

\section{Competing interests}

The authors declare that they have no competing interests.

\section{References}

1. Mikkelsen LH, Larsen AC, von Buchwald C, Drzewiecki KT, Prause JU and Heegaard S: Mucosal malignant melanoma-a clinical, oncological, pathological and genetic survey. APMIS 124: 475-486, 2016.

2. Yde SS, Sjoegren P, Heje M and Stolle LB: Mucosal melanoma: A literature review. Curr Oncol Rep 20: 28, 2018.

3. Nishie W: Collagen XVII processing and blistering skin diseases. Acta Derm Venereol 12: adv00054, 2020.

4. Krenacs T, Kiszner G, Stelkovics E, Balla P, Teleki I, Nemeth I, Varga E, Korom I, Barbai T, Plotar V, et al: Collagen XVII is expressed in malignant but not in benign melanocytic tumors and it can mediate antibody induced melanoma apoptosis. Histochem Cell Biol 138: 653-667, 2012.

5. Hwang BJ, Zhang Y, Brozowski JM, Liu Z, Burette S, Lough K, Smith CC, Shan Y, Chen J, Li N, et al: The dysfunction of BP180/collagen XVII in keratinocytes promotes melanoma progression Oncogene 38: 7491-7503, 2019.

6. Sawabe M, Arai T, Kasahara I, Esaki Y, Nakahara K, Hosoi T, Orimo H, Takubo K, Murayama S and Tanaka N; Tokyo Metropolitan Geriatric Medical Center; Japan Science and Technology Agency: Developments of geriatric autopsy database and Internet-based database of Japanese single nucleotide polymorphisms for geriatric research (JG-SNP). Mech Ageing Dev 125: 547-552, 2004.

7. Yamada M, Sato N, Ikeda S, Arai T, Sawabe M, Mori S, Yamada Y, Muramatsu M and Tanaka M: Association of the chromodomain helicase DNA-binding protein 4 (CHD4) missense variation p.D140E with cancer: Potential interaction with smoking. Genes Chromosomes Cancer 54: 122-128, 2015.

8. Eguchi $\mathrm{H}$ and Okazaki Y: Next-generation sequencing for genetic diagnosis of hereditary colorectal cancer and polyposis syndrome. In: Recent Advances in the Treatment of Colorectal Cancer. Ishida H (eds). Springer, pp115-125, 2019.

9. Tadaka S, Hishinuma E, Komaki S, Motoike IN, Kawashima J, Saigusa D, Inoue J, Takayama J, Okamura Y, Aoki Y, et al: jMorp updates in 2020: Large enhancement of multi-omics data resources on the general Japanese population. Nucleic Acids Res 49: D536-D544, 2021.

10. Adzhubei I, Jordan DM and Sunyaev SR: Predicting functional effect of human missense mutations using PolyPhen-2. Curr Protoc Hum Genet Chapter 7: Unit7.20, 2013.

11. Peng J, Han F, Yang T, Sun J, Guan W and Guo X: Primary malignant melanoma of the lung: A case report and literature review. Medicine (Baltimore) 96: e8772, 2017.

12. Tomizuka T, Namikawa $\mathrm{K}$ and Higashi T: Characteristics of melanoma in Japan: A nationwide registry analysis 2011-2013. Melanoma Res 27: 492-497, 2017.

13. Carvajal RD, Spencer SA and Lydiatt W: Mucosal melanoma: A clinically and biologically unique disease entity. J Natl Compr Canc Netw 10: 345-356, 2012.

14. Kamaguchi $\mathrm{M}$ and Iwata $\mathrm{H}$ : The diagnosis and blistering mechanisms of mucous membrane pemphigoid. Front Immunol 24: 34, 2019.

15. Pogorzelska-Dyrbus J and Szepietowski JC: Adhesion molecules in non-melanoma skin cancers: A comprehensive review. In Vivo 35: 1327-1336, 2021.

16. Tanimura S, Tadokoro Y,Inomata K,BinhNT,NishieW, YamazakiS, Nakauchi H, Tanaka Y, McMillan JR, Sawamura D, et al: Hair follicle stem cells provide a functional niche for melanocyte stem cells. Cell Stem Cell 8: 177-187, 2011.
17. Liu N, Matsumura H, Kato T, Ichinose S, Takada A, Namiki T, Asakawa K, Morinaga H, Mohri Y, De Arcangelis A, et al: Stem cell competition orchestrates skin homeostasis and ageing. Nature 568: 344-350, 2019.

18. Natsuga K, Watanabe M, Nishie $W$ and Shimizu H: Life before and beyond blistering: The role of collagen XVII in epidermal physiology. Exp Dermatol 28: 1135-1141, 2019.

19. Vishwakarma $M$ and Piddini E: Outcompeting cancer. Nat Rev Cancer 20: 187-198, 2020.

20. Ferrari AJ, Drapkin R and Gogna R: Cell fitness: More than push-ups. Int J Mol Sci 22: 518, 2021.

21. Yodsurang V, Tanikawa C, Miyamoto T, Lo PHY, Hirata M and Matsuda K: Identification of a novel p53 target, COL17A1, that inhibits breast cancer cell migration and invasion. Oncotarget 8 : 55790-55803, 2017.

22. Lothong M, Sakares W, Rojsitthisak P, Tanikawa C, Matsuda K and Yodsurang V: Collagen XVII inhibits breast cancer cell proliferation and growth through deactivation of the AKT/mTOR signaling pathway. PLoS One 16: e0255179, 2021.

23. Kozawa K, Sekai M, Ohba K, Ito S, Sako H, Maruyama T, Kakeno M, Shirai T, Kuromiya K, Kamasaki T, et al: The CD44/COL17A1 pathway promotes the formation of multilayered, transformed epithelia. Curr Biol 26: 3086-3097.e7, 2021.

24. Martin SK and Wood RD: DNA polymerase $\zeta$ in DNA replication and repair. Nucleic Acids Res 19: 8348-8361, 2019.

25. Chae YK, Anker JF, Carneiro BA, Chandra S, Kaplan J, Kalyan A, Santa-Maria CA, Platanias LC and Giles FJ: Genomic landscape of DNA repair genes in cancer. Oncotarget 26: 23312-23321, 2016

26. Song L, McNeil EM, Ritchie AM, Astell KR, Gourley C and Melton DW: Melanoma cells replicate through chemotherapy by reducing levels of key homologous recombination protein RAD51 and increasing expression of translesion synthesis DNA polymerase $\zeta$. BMC Cancer 18: 864, 2017.

27. Arivazhagan R, Lee J, Bayarsaikhan D, Kwak P, Son M, Byun K, Salekdeh GH and Lee B: MicroRNA-340 inhibits the proliferation and promotes the apoptosis of colon cancer cells by modulating REV3L. Oncotarget 26: 5155-5168, 2017.

28. Strong AM, Setaluri V and Spiegelman VS: MicroRNA-340 as a modulator of RAS-RAF-MAPK signaling in melanoma. Arch Biochem Biophys 1: 118-124, 2014.

29. Julius MA, Schelbert B, Hsu W, Fitzpatrick E, Jho E, Fagotto F, Costantini F and Kitajewski J: Domains of axin and disheveled required for interaction and function in wnt signaling. Biochem Biophys Res Commun 276: 1162-1169, 2000.

30. Ikeda S, Kishida S, Yamamoto H, Murai H, Koyama S and Kikuchi A: Axin, a n9gative regulator of the Wnt signaling pathway, forms a complex with GSK-3beta and beta-catenin and promotes GSK-3beta-dependent phosphorylation of beta-catenin. EMBO J 17: 1371-1384, 1998.

31. Castiglia D, Bernardini S, Alvino E, Pagani E, Luca ND, Falcinelli S, Pacchiarotti A, Bonmassar E, Zambruno G and D'Atri S: Concomitant activation of Wnt pathway and loss of mismatch repair function in human melanoma. Genes Chromosomes Cancer 47: 614-624, 2008

32. Smith AA, Li J, Liu B, Hunter D, Pyles M, Gillette M, Dhamdhere GR, Abo A, Oro A and Helms JA: Activating hair follicle stem cells via R-spondin2 to stimulate hair growth. J Invest Dermatol 136: 1549-1558, 2016.

33. Du Y, Shao H, Moller M, Prokupets R, Tse YT and Liu ZJ: Intracellular notch1 signaling in cancer-associated fibroblasts dictates the plasticity and stemness of melanoma stem/initiating cells. Stem Cells 37: 865-875, 2019.

34. Chen J, Wu F, Shi Y, Yang D, Xu M, Lai Y and Liu Y: Identification of key candidate genes involved in melanoma metastasis. Mol Med Rep 20: 903-914, 2019.

This work is licensed under a Creative Commons Attribution-NonCommercial-NoDerivatives 4.0 International (CC BY-NC-ND 4.0) License. 